\title{
VENUS BOWSHOCK PRECURSOR
}

\author{
Ernest G. Fontheim* and Larry H. Brace** \\ *Space Physics Research Laboratory, Department of Atmospheric, Oceanic \\ and Space Sciences, The University of Michigan, Ann Arbor, MI 48109, \\ U.S.A. \\ ** Laboratory for Atmospheres, NASA Goddard Space Flight Center. \\ Greenbelt, MD 2077I, U.S.A.
}

\section{ABSTRACT}

Significant ion and electron flux enhancements immediately upstream of the Venus bowshock have been observed by the Electron Temperature Probe on the Pioneer Venus Orbiter. It is shown that mass loading of the solar wind by oxygen ions (without the effect of MHD turbulence) accounts for only about $3.5 \%$ of the observed effect. Finally, evidence is presented that turbulence may play a nonnegligible role in the ion pickup process in front of the Venus bowshock.

\section{INTRODUCTION}

Recent observations by the Electron Temperature Probe on the Pioneer Venus Orbiter indicate enhanced electron and ion currents immediately upstream of the Venus bowshock /1,2,3/. In particular, Brace et al. $3 /$ have shown that the precursor observations are consistent with the hypothesis that they are caused by the presence of hot ions upstream of the shock. Mass-loading of the upstream particle flux will be examined in this paper as a potential mechanism which could contribute to the observed electron and ion flux enhancements.

Venus is known to have a hot oxygen corona $/ 4,5 /$ resulting in an extended atmosphere. Oxygen is the dominant neutral gas species on the dayside out to distances of about $3000 \mathrm{~km}$ during solar maximum conditions $/ 6 /$. The bowshock is located at an altitude of only about $2300 \mathrm{~km}$ at the subsolar point during solar maximum $/ 7 /$ which is inside the region of this hot oxygen corona. The oxygen atoms are ionized by either photoionization or charge exchange with solar wind protons. The $\mathrm{O}^{+}$ions are then picked up by the solar wind, thus, mass loading the flow. As a result of this energy transfer from the solar wind protons to the $\mathrm{O}^{+}$ions, the bulk flow velocity decreases. This decrease in the solar wind flow velocity as well as the addition of $\mathrm{O}^{+}$result in an increase in the ion (and electron) number density of the mass loaded solar wind.

\section{DISCUSSION OF THEORY AND UNDERLYING ASSUMPTIONS}

The calculations presented here make use of earlier work on the solar wind interaction with comets $/ 8,9 /$ with appropriate modifications for the situation at Venus. The purpose of this paper is to establish qualitatively the feasibility of mass loading as a cause of the precursor therefore the calculations are simplified as much as possible. The calculations described in this paper are based on the following assumptions: 1) One-dimensional flow, 2) magnetic field perpendicular to the solar wind velocity vector, 3) no diffusion in either pitch angle or energy of the implanted $\mathrm{O}^{+}$ions, and 4) conservation of the magnetic moment (first invariant) of the implanted ions. The total $\mathrm{O}^{+}$production rate is given by the expression

$$
R_{T}=R_{p h}+R_{c e}=n_{o}\left[\Sigma+\sigma_{c e}\left(E_{p}\right) \Phi_{S w}\right]
$$

where $R_{\mathrm{ph}}$ and $\mathrm{R}_{c e}$ are the photoionization and proton charge exchange rates of $\mathrm{O}$, respectively and

$$
\Sigma \equiv \int_{E_{1} /}^{\infty} \sigma p(v) \Phi(v) d v,
$$

and where $n_{0}$ is the hot atomic oxygen density, $E_{i}$ is the oxygen ionization potential, $h$ is Planck's constant, $\sigma_{p}(v)$ is the photoionization cross section of oxygen for photons of frequency $v, \sigma_{c e}\left(E_{p}\right)$ is the charge exchange cross section of $O$ with solar wind protons of energy $E_{p}$ $\left(=2 \times 10^{-9} \mathrm{erg}\right.$ for a typical solar wind velocity of $450 \mathrm{~km} / \mathrm{s}$ at solar maximum), and $\Phi_{s w}=n_{\infty} u_{\infty}$ is the unperturbed solar wind proton flux. $\mathrm{R}_{\mathrm{T}}$ is considered to be only a function of the altitude. The two cross sections and fluxes are considered to be constant in space and time. The altitude dependence of $R_{\mathrm{T}}$ is a result of the altitude dependence of $n_{0}$. The calculations presented here are based on separate continuity equations, one for the implanted $\mathrm{O}^{+}$ions and one for the contaminated solar wind, and a momentum equation for the contaminated solar wind. The solution to these equations is $19,10 /$

$$
\begin{gathered}
\hat{\rho} \hat{u}=1+\left(m_{i} / \rho_{\infty} u_{\infty}\right)\left(\Sigma+\sigma_{c e} \Phi_{s w}\right) \int_{x}^{x} n_{0}\left(x^{\prime}\right) d x^{\prime} \\
\hat{\rho}=2 \hat{\rho} \hat{u}\left[1-(1-3 \hat{\rho} \hat{u} / 4)^{1 / 2}\right]
\end{gathered}
$$




$$
\begin{gathered}
\hat{u}=(2 / 3 \hat{\rho} \hat{u})\left[1+(1-3 \hat{p} \hat{u} / 4)^{1 / 2}\right] \\
\rho_{i} u f(u, \mu)=\frac{4\left(\hat{\rho}^{1 / 3}-0.5\right)}{9 \hat{\rho}^{5 / 3} \mu_{\infty}} \rho_{\infty} u_{\infty} \Theta(\mu-\mu) \Theta\left(\mu-\mu_{\infty} \hat{u}^{3}\right)
\end{gathered}
$$

where $\hat{p}, \hat{u}$. and $\hat{\mu}$ are the solar wind parameters normalized to their unperturbed values $\rho_{\infty}, u_{\infty}, \mu_{\infty}$ and where $\mu_{\infty}=m_{i} u_{\infty}{ }^{2} / 2 B_{\infty}$ and $\Theta(x)=1$ for $x>0$ and $\Theta(x)=0$ for $x<0$. Hence, under the assumption of the conservation of the first invariant, the magnetic moment of the implanted ions is restricted to the range

$$
\mu_{\infty} \hat{\mathrm{u}}^{3}<\mu<\mu_{\infty}
$$

The total mass density of the implanted ions is therefore

$$
\int_{\hat{u}_{\mu}^{3}}^{\mu} \rho_{i} f(u, \mu) d \mu=(1 / 3) \rho_{\infty} \hat{u}^{-1}\left(-3+4 \hat{u}^{-1}-\hat{u}^{-2}\right)
$$

\section{RESULTS AND DISCUSSION}

The hot atomic oxygen density profile for solar maximum conditions was obtained from ultraviolet spectrometer measurements made onboard the Pioneer Venus Otbiter $/ 5 /$. This results in a column density, defined by the integral in equation (3), of $2.4 \times 10^{11} \mathrm{~cm}^{-2}$. The atomic oxygen photoionization rate $\Sigma$ of $4.5 \times 10^{-7} \mathrm{~s}^{-1}$ at $1 \mathrm{AU}$ for solar maximum conditions was obtained from Table 7.19 of Banks and Kockarts $/ 11$, which gives a value of $8.6 \times 10^{-7} \mathrm{~s}^{-1}$ at the Venus orbit of 0.723 AU. The charge exchange cross section for neutral oxygen atoms with solar wind protons has been taken to be $1.5 \times 10^{-15} \mathrm{~cm}^{2}$ (equation 9.88 of $/ 11 /$ ). The unperturbed solar wind parameters at 1 $\mathrm{AU}$ are taken to be $\mathrm{n}_{\infty}=6 \mathrm{~cm}^{-3}, \mathrm{u}_{\infty}=4.5 \times 10^{7} \mathrm{~cm} / \mathrm{s}$ which translates into a flux of $5.2 \times 10^{8} \mathrm{~cm}^{-2} \mathrm{~s}^{-1}$ at $0.723 \mathrm{AU}$.

Upon substituting these values into equation (3) one obtains

$$
\rho u=\left(1+1.4 \times 10^{-2}\right) p_{\infty} u_{\infty} \text {. }
$$

which represents a $1.4 \%$ increase in the mass flow. If this solution is substituted into equation (4), one obtains $\hat{p}=1+3.5 \times 10^{-2}$ which represents a $3.5 \%$ increase in the mass density. This increase contains the combined effects of the addition of implanted ions and the increase in number density of the solar wind protons caused by the decrease in the flow velocity. The latter, of course, is a consequence of mass loading and can be obtained from equation (5),

$$
\hat{\mathbf{u}}=1 \cdot 2.1 \times 10^{-2} \text {. }
$$

Hence, the mass loading results in a slowdown of $2.1 \%$ in the solar wind speed in front of the Venus bowshock. We are now in a position to calculate the mass density of the implanted $\mathrm{O}^{+}$ions and the increase in the number density of $\mathrm{H}^{+}$ions. Using the parameters listed above, the mass density of implanted $\mathrm{O}^{+}$ions is obtained from equation (7)

$$
\rho_{i} \int_{\substack{\mu \\ \hat{u}^{3}}}^{\mu} f(u, \mu) d \mu=1.4 \times 10^{-2} \rho_{\infty} .
$$

Thus, a $1.4 \%$ mass density increase is due to the addition of $\mathrm{O}^{+}$to the flow. The remaining $2.1 \%$ increase (to give a total mass density increase of $3.5 \%$ ) is due to the density increase in solar wind protons.

These results are of course based on the simplifying assumptions stated in the preceding section. Recently a number of papers was published $/ 12,13,14$ / presenting more general theories than the one on which this paper is based. In particular, these authors include effects due to microscopic processes (waves and instabilities). These effects include ion pickup, pitch angle scattering of the pickup ions, and solar wind flow deceleration. Therefore ion pickup occurs not only due to the perpendicular component of ion velocity to the magnetic field but also due to its parallel component. Omidi and Winske /13/ have carried out extensive modeling of mass loading for several values of the angle between the solar wind velocity and the magnetic field (cone angle). They show that for cone angles near $90^{\circ}$ the dominant mass loading pocess is the coupling through macroscopic fields (i.e., $\mathbf{E} \times \mathbf{B}$ ) on which our results are based. Hence, as the cone angle becomes smaller, mass loading through macroscopic fields becomes less efficient, while microscopic processes become more important as the pickup process. Sagdeev et al. /15/ have recently shown that mass loading of the solar wind to distances beyond the cometary bowshock excites Alfven turbulence the amplitude of which is of the order of the interplanetary magnetic field. Such turbulence has indeed been observed near comet Halley (including the region outside the bowshock) not only in the magnetic field but also in the electron density data $/ 16,17,18,19,20 \%$. A preliminary comparison of Pioneer Venus magnetic field data $/ 21 /$ and Langmuir probe electron current data of several orbits reveals an evident correlation between magnetic field fluctuations and Langmuir probe electron density data. An example of such a correlation is presented in Figure 1. The ordinate of the lower panel presents the ratio of the square root of the trace of the variance matrix to the magnetic field magnitude. The variance matrix is defined by the expression

$$
\left.\left.R_{i j}=N^{-1} \Sigma_{k}\left[B^{(k)}\right)_{i}<B_{i}>\right]\left[B^{(k)}\right)_{j}<B_{j}>\right] .
$$

where the values of $B^{(k)}$ are 13-sec averages of $B_{m}$ and the upper index (k) identifies successive values of this average. The sum over $k$ is taken over a $130 \mathrm{~s}$ interval, and the averages $<>$ are taken over the same $130 \mathrm{~s}$ interval. For each $130 \mathrm{~s}$ interval a variance matrix is calculated. In this way, any spin modulation of the magnetic field due to the satellite spin ( $13 \mathrm{~s}$ period ) is taken out. The trace of this 


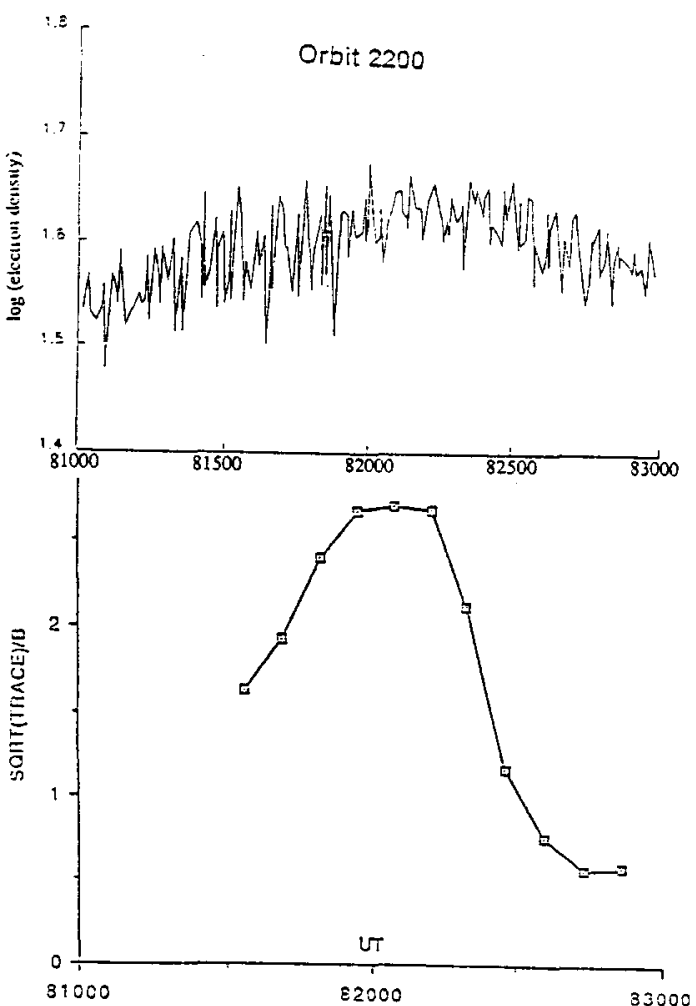

Fig. 1: Upper Panel: Electron density variation in the Venus bowshock precursor. Lower Panel: Square root of the trace of the magnetic field variance matrix normalized by the magnitude of the field

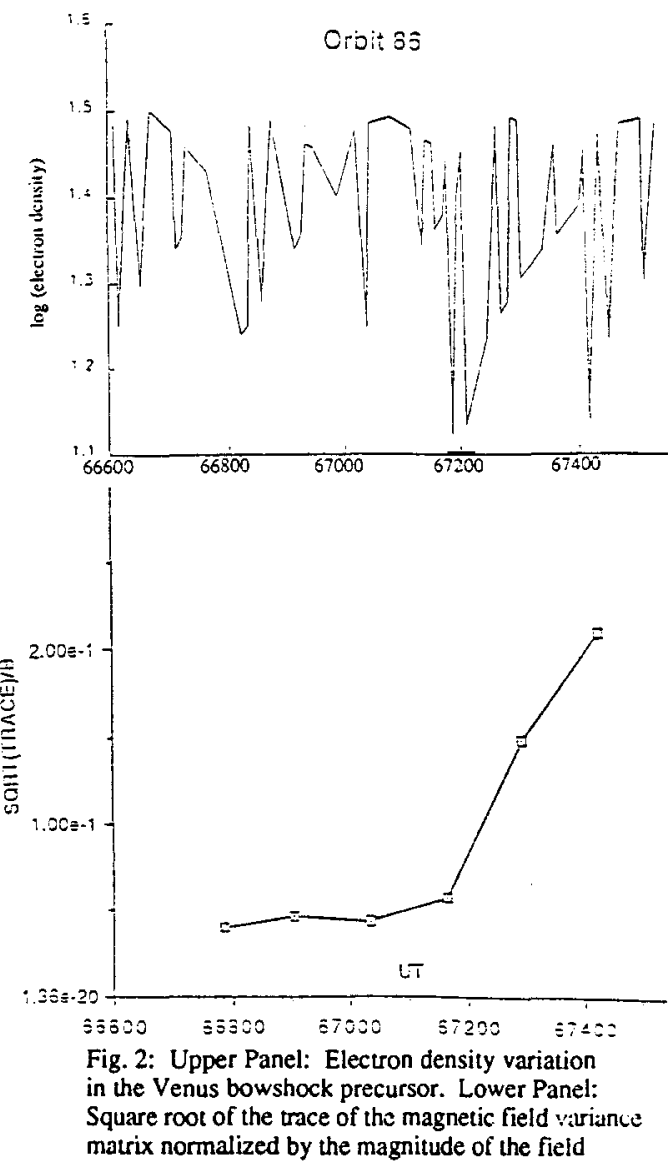

matrix is a measure of the power in the MHD waves. As Figure 1 indicates, the magnitude of the fluctuations correlates with the density enhancements (upper panel), and it considerably exceeds the value of the magnetic field iself. Figure 2 is an example of a case which shows neither a precursor density enhancement nor any magnetic turbulence. The lower panel shows that the variance is in the noise level of the magnetic field. The scenario of this mechanism is that MHD turbulence is excited by mass loading because the mass-loaded flow is highly anisotropic and therefore unstable. The MHD turbulence gives rise to a collective pickup of the newly created ions in the direction parallel to the magnetic field. This process represents an additional mechanism of momentum transfer from the solar wind flow to the pickup ions by way of MHD turbulence whereby the turbulence absorbs any momentum difference. Associated with this momentum transfer is an additional solar wind-slowdown and therefore a potential source for a density increase.

Another effect of pitch angle scattering is a change in the value of the specific heat ratio $\gamma$ which has been taken to be 2 in the calculations presented here. If pitch angle scautering is included, $\gamma$ should be taken as $5 / 3$ (appropriate for three degrees of freedom). The effect of a decreased value of $\gamma$ can be obtained from the equation for the deceleration of the mass-loaded solar wind $/ 8 /$

$$
\mathrm{du} / \mathrm{dx}=(\gamma+1) \mathrm{u}^{2} \rho_{\mathrm{n}} / 2\left(\gamma \cdot \rho \mathrm{u}^{2}\right) \tau \text {. }
$$

It is easily seen that $\mathrm{du} / \mathrm{dx}$ decreases with increasing $\gamma$ or - in ocher words - the solar wind deceleration increases with increasing $\gamma$. Hence, the reduction in the value of $\gamma$ leads to a reduced solar wind deceleration which in tum implies a reduced plasma density increase. The inclusion of pitch angle scattering in our calculations would have resulted in a smaller density enhancement for two reasons, a redistribution of the pickup ions to pitch angles other than $90^{\circ}$ and a reduction in the specific heat ratio $\gamma$. Our results therefore represent an upper bound of the electron density enhancement due to mass loading of the upstream solar wind by $\mathrm{O}^{+}$ions.

The description of the pickup ions is based on the assumption of a small gyroradius compared to the dimensions of the interaction region under consideration. In the present case this is the thickness of the precursor. The gyroradius of $\mathrm{O}^{+}$ions of near solar wind speed perpendicular to the IMF is of the order of several thousand $\mathrm{km}$ which is about the magnitude of the precursor thickness. Phillips et al. 22/ have recently shown that finite gyroradius effects are significant in the Venus magnetosheath due to the cylindrical asymmetry of the motional electric field in the magnetosheach between the two Venus hemispheres. In the unshocked solar wind, finite gyroradius effects will result in a redistribution of the pickup ions. The mass loading locations, on the other hand, are distributed according to the locations where the oxygen ionizations occur which in turn follow the distribution of neutral oxygen at a given radial distance from Venus. The solar wind decelerating force field acts at the mass loading locations and therefore also follows the neutral oxygen distribution. Therefore the plasma density increase due to mass loading is not affected by finite gyroradius effects, and, as shown above, this is the major part of the total density increase.

\section{CONCLUSIONS}

The calculations presented here show that mass loading of the solar wind by $O^{+}$will result in a number density increase of at most $2.2 \%$. If the observed $30 \%$ electron flux enhancement in the precursor is assumed to be entirely due to a corresponding density enhancement in the upstream solar wind, it is of interest to calculate the minimum required atomic oxygen column density which is necessary to obtain such a density change. This turns out to be a column density of $3.2 \times 10^{12} \mathrm{~cm}^{-2}$ which is about one order of magnitude larger than the 
column density used in the above calculations. No atomic oxygen column density of that magnitude has been observed by Pioneer Venus 23/. Therefore these calculations indicate that mass loading of the solar wind flow in front of the bowshock due to coupling by macroscopic fields cannot be the sole mechanism responsible for the observed enhancements in the electron and ion fluxes. Therefore the observed increases in the Langmuir probe collection current upstream of the Venus bowshock must either be the result of some other physical process in the preshocked solar wind flow, like the above mentioned Alfven turbulence, or the result of secondary electron cmission from the spacecraft and/or the collector $3 /$.

\section{ACKNOWLEDGMENTS}

This research was supported by NASA Grants NAG 5-844 and NAG 2.518 to the University of Michigan. The authors are grateful to T.C. Cravens for helpful suggestions and to A.F. Nagy for his critical reading of the manuscript. The authors also gratefully acknowledge many useful conversations with J.G. Luhmann, C.T. Russell, and F.L. Scarf.

\section{REFERENCES}

1. L.H. Brace, S.A. Curtis, C.T. Russell, and F.L. Scarf, A precursor to the Venus bowshock, Irans, Am, Geophys, U, (EOS) 66, 294 (1985)

2. L.H. Brace, Additional Pioneer Venus observations of the precursor to the Venus bowshock. Irans. Am. Geophys. U. (EOS) 68, 342 (1987)

3. L.H. Brace, R.F. Theiss, S.A. Curtis, and L.W. Parker, A precursor to the Venus bow shock, L. Geophys. Res, 93, 12735 (1988)

4. A.F. Nagy, T.E. Cravens, J.-H. Yee, and A.I.F. Stewart, Hot oxygen atoms in the upper atmosphere of Venus, Geophys, Res, Lell. $8,629(1981)$

5. LJ. Paxton, Atomic Carbon in the Venus Thermosphere: Observations and Theory Thesis, Department of Astro-Geophysics, University of Colorado (1983) (Fig. B-8)

6. A.J. Kliore, V.I. Moroz, G.M. Keating, ed's., The Venus International Reference Aumosphere, Pergamon Press, Oxford, U.K., Chapter IV.4 (1986)

7. J.G. Luhmann, The solar wind interaction with Venus, Space Sci. Rev 44, 241 (1986)

8. M.K. Wallis, and R.S.B. Ong, Strongly-cooled ionizing plasma flows with application to Venus, Planet. Space Sci. 23, 713 (1975)

9. A.A. Galeyev, T.E. Cravens, and T.I. Gombosi, Solar wind stagnation near comets, Astrophys. J 289, 807 (1985)

10. L. Biermann, B. Brosowski, and H.U. Schmidt, The interaction of the solar wind with a comet, Solar Phys, 1, 254 (1967)

11. P.M. Banks, and G. Kockarts, Aeronomy Part A. Academic Press, New York and London (1973)

12. A.A. Galeyev, Theory and observations of solar wind/cometary plasma interaction processes. Proceedings of the 20th ESLAB Symposium on the Exploration of Halley's Comet, Eur. Space Azency Spec. Publ. ESA SP 250 1, 3 (1986)

13. N. Omidi and D. Winske, A kinetic study of solar wind mass loading and cometary bow shocks, L. Geophys. Res, 92, 13409 (1987)

14. T.I. Gombosi, Preshock region acceleration of implanted cometary $\mathrm{H}^{+}$and $\mathrm{O}^{+}$, L.Geophys. Res, 93, 35 (1988)

15. R.Z. Sagdeyev, V.D. Shapiro, V.I. Shevchenko, and K. Szegס, MHD turbulence in the solar wind-comet interaction region, Geophys. Res.Lell. 13, 85 (1986)

16. W. Riedler, K. Schwingenschuh, Ye.G. Yeroshenko, V.A. Styashkin, and C.T. Russell, Magnetic field observations in comet Halley's coma, Nature 321, 288 (1986)

17. F.M. Neubauer, Giotto magnetic field results on the magnetic field pile-up region and the cavity boundaries, Proceedings of the 20th ESLAB Symposium on the Exploration of Halley's Comet, Eur Space_Agency Spec. Publ. ESA SP 250 1, 35 (1986)

18. F.M. Neubauer, K.H. Glassmeier, M. Pohl, J. Raeder, M.H. Acuña, L.F. Burlaga, N.F. Ness, G. Musmann, F. Mariani, M.K. Wallis, E. Ungstrupp, and H.U. Schmidt, First results from the Giotto magnetometer experiment at comet Halley, Nature 321, 352 (1986)

19. H. Rème, J.A. Sauvaud, C. dUston, F. Cotin, A. Cros, K.A. Anderson, C.W. Carison, D.W. Curtis, R.P. Lin, D.A. Mendis, A. Korth, and A.K. Richter, Comet Halley-solar wind interaction from electron measurements aboard Giotto. Nature 321,349 (1986a)

20. H. Rème, J.A. Sauvaud, C. dUston, A. Cros, K.A. Anderson, C.W. Carlson, D.W. Curtis, R.P. Lin, A. Korth, A.K. Richter, and D.A. Mendis, General features of the comet Halley-solar wind interaction from plasma measurements, Proceedings of the 20th ESLAB Symposium on the Exploration of Halley's Comet, Eur. Space Agency Spec. Publ. ESA SP 250 1, 29 (1986b)

21. C.T. Russell, private communication (1988)

22. J.L. Phillips, J.G. Luhmann, C.T. Russell, and K.R. Moore, Finite Larmor radius effect on ion pickup at Venus J.Geophys. Res. $92,9920(1987)$

23. L.J. Paxton, private communication (1988) 